\title{
KONFERENZBAND DER JUNGEN SLAVISTIK
}

(Nelli RitTer \& MARTIN HeNZELMANN, HRSG., LINGUISTISCHE BEITRÄGE ZUR

SLAVISTIK. XXVIII. JUNGSLAVISTINNEN-TREFFEN, 18. BIS 20. SEPTEMBER 2019 IN

Hamburg, Specimina PHILOlogiae SlaVicae, 206, Berlin, Bern, Wien et AL.:

VERLAG PETER LANG, 2021, 292 SEITEN, ISBN: 978-3-631-86056-4, 69,95€, CHF 81,00)

Boryan Yanev, PhD, Philologische Fakultät, Universität „Paisii

Hilendarski" Plovdiv, byanev@uni-plovdiv.bg

DOI: 10.31902/fII.36.2021.21

Der hier zu rezensierende Sammelband präsentiert die Ergebnisse des XXVIII. "JungslavistInnen-Treffens", das im September 2019 an der Universität Hamburg stattfand. Dieser junge Kreis von Forschern aus dem deutschsprachigen Traum trifft sich regelmäßig und man kann aus der Reihe „Specimina philologiae Slavicae“ (Verlag Peter Lang) ablesen, dass schon etliche Bände publiziert wurden. Es ist erfreulich, dass die Slavistik in Deutschland über solch eine selbstorganisierte Infrastruktur verfügt.

Kommen wir nun zu den Artikeln, die sich über ein weitreichendes sprachwissenschaftliches Spektrum erstrecken. Sprachhistorische und gegenwärtige Standpunkte der Slavistik sind im Buch nachzulesen. Es sind also aktuelle Forschungsergebnisse von elf slavistischen Nachwuchswissenschaftlern in Deutschland abgedruckt.

Im Folgenden werde ich kurz die Inhalte der Texte vorstellen. Ich werde zudem meine Einschätzung zu den Arbeiten vor einem sprachwissenschaftlichen Hintergrund abgeben und die Frage reflektieren, welchen Beitrag diese Publikation zur Slavistik leistet.

Beginnen möchte ich mit dem ersten Text von Ruben Biewald. Er trägt den Titel „Stil oder Grammatik? Methodische Überlegungen zu einer Untersuchung der ukrainischen Bibelübersetzung" (S. 9-21). Der Autor erklärt, dass die Übersetzung der Bibel ins Ukrainische im 19. Jahrhundert nicht nur der Verständlichkeit des Texts dienen, sondern einen Beitrag zur Schaffung einer neuen ukrainischen Schrifttradition leisten sollte. Eine Übersetzung des Bibeltexts konnte dabei nicht ohne Anschluss an die bisherige Schrifttradition gelingen, und so erfolgte die Übersetzung ausgerichtet der mündlichen ukrainischen Sprache. Autoren wie Kuliš und Puljuj und ihre Veröffentlichungen Anfang des 19. 
Jahrhunderts markierten den Beginn der Herausbildung eines eigenen konfessionellen Stils im Ukrainischen, was etwa anhand der Funktionen der Partizipien und deren Übersetzungstypologie in die jeweiligen modernen ostslavischen Sprachen gezeigt wird. Das ist bemerkenswert, jedoch ist die Rolle der Bibelsprache und ihr Einfluss auf die Konsolidierung moderner Schriftsprachen besonders im orthodoxen Kulturraum natürlich ein weit verbreitetes Phänomen.

Der nächste Beitrag (S. 23-50) stammt von Christina Clasmeier und lautet „Zur Wortfolge polnischer Adjektiv-Substantiv-Konstruktionen in der Sprachverarbeitung - ein Satzwiederholungsexperiment“. Die Autorin stellt dar, dass in polnischen Nominalphrasen das attributive Adjektiv sowohl vor als auch nach dem Substantiv positioniert sein kann, wenngleich die Reihenfolge nicht willkürlich ist. Doch worin besteht dann die Regelmäßigkeit? Auf diese Frage versucht sie eine Antwort zu geben, indem sie Probanden mit einer sogenannten Sentence Repetition Task konfrontiert und die Ergebnisse systematisiert. Diese experimentelle Herangehensweise ist sicher interessant, hat aber den Nachteil, dass dadurch keine Beweise erbracht werden können, so dass die Ergebnisse vor allem von den Probanden abhängen und deshalb variabel sein dürften.

Der Text des Mitherausgebers Martin Henzelmann widmet sich dem Thema „Das Bunjewatzische in Serbien nach 1990: Innovationen und Kontroversen" (S. 51-75). Darin werden die Schritte besprochen, die die bunjewatzische Minderheit in der serbischen Vojvodina unternommen hat, um ihrer Sprache einen schriftlichen Ausdruck zu verleihen. Dass die Varietäten, die die Bunjewatzen sprechen, praktisch alle Merkmale mit den Sprachen teilt, die früher unter dem Terminus "Serbokroatisch" subsummiert wurden, hindert sie offenbar nicht an ihren Aktivitäten. Man mag rätseln, ob diese nun als Wunschdenken oder als Genugtuung klassifiziert werden sollten. Ungeachtet dessen bearbeitet der Mitherausgeber ein originelles Thema, welches die Frage nach Sprache und Identität, aber auch nach eigenem Anspruch und fremder Beanspruchung der Minderheit stellt. In der internationalen Slavistik ist die Problematik noch sehr wenig präsent und sollte zusätzliche Beachtung finden.

Nicolas Jansen ist der Autor des umfangreichsten Textes dieses Sammelbandes. Auf den Seiten 77 bis 139 analysiert er „Das Ende des Alec Holowka: Ein Todesfall aus russisch- und englischsprachiger medialer Perspektive“. Besagter Holowka dürfte in der Slavistik kaum jemandem bekannt gewesen sein, denn es handelte sich um einen Entwickler von Computerspielen, der unter merkwürdigen Umständen sein Leben ließ. Jansen untersucht eine Diskussion, die sich durch Zoë 
Quinn - sie entstammt ebenfalls der Spielentwicklerszene - entfachte. Sie warf Holowka schweres Fehlverhalten vor. Die daraus resultierenden Kommentare in Foren werden $\mathrm{zu}$ einem eigenen und sehr umfangreichen Korpus mit russischen und englischen Daten in akribischer Arbeit zusammengestellt. Es wird auf dieser Grundlage kontrastiv untersucht, welche metasprachlichen Komponenten in der Beurteilung des Sachverhalts auffällig sind.

Interessant ist auch der Artikel, den Irenäus Kulik auf den Seiten 141 bis 159 vorstellt. Er untersucht „Populäre Musik als sprachwissenschaftliche Quellengattung? Das Beispiel Schlesisch“. Seiner Einschätzung nach ist populäre Musik noch keine hinreichende Quelle sprachwissenschaftlicher Untersuchungen. Dieser Ansicht kann man mit Hinblick auf sein Beispiel - Heavy Metal (sic!) - sicher uneingeschränkt folgen, noch dazu, wenn es um das Schlesische geht. Der Autor stellt ein eigenes Korpus zusammen und beleuchtet die darin vorkommende Graphemik, Lexik, Phonologie, Morphologie und Syntax, die allesamt Eigentümlichkeiten des Schlesischen aufweisen. Auch zu diesem Text ist zu sagen, dass er einen innovativen Ansatz verfolgt und mit belegbaren Daten arbeitet.

Alisa Müller (S. 161-188) stellt anschließend die Frage: „Haben das Russische und das Belarussische in der Linguistic Landscape von Minsk unterschiedliche Funktionen?" In der Linguistic Landscape konzentriert man sich sehr häufig auf die Verbreitung unterschiedlicher Sprachen im öffentlichen Raum. Die Autorin wagt darüber hinaus den Versuch einer funktionalen Klassifizierung des Weißrussischen und des Russischen anhand von Aufschriften, die sie auf einem zentralen Boulevard in Minsk identifizierte, so dass auch sie ein eigenes Korpus erstellt und Empirie verwertet. In ganz Weißrussland finden wir zwar bekanntlich nahezu alle Aufschriften in russischer oder belarussischer Sprache, was Müller hier vorstellt ist aber sehr wichtig, denn wir wissen auch, dass es sich um eine themenabhängige und nicht willkürliche Verwendung der beiden Sprachen im öffentlichen Raum handelt.

Hagen Pitsch untersucht „Die Aorist/Imperfekt-Distinktion im Bulgarischen: Form, Bedeutung, Markiertheit“ (S. 189-208). Seine Studie hinterfragt die Signifikanz des bulgarischen Aorists und des Imperfekts. Er orientiert sich bei seiner Analyse an vorherigen Studien, die freilich sehr zahlreich sind, und stellt heraus, dass das Imperfekt eine offene Zeitspanne für weitreichende und irreale Situationen markiert. Der Aorist dient demnach als unmarkierte Zeitform der einfachen Vergangenheit. Gegen diese These ist nichts einzuwenden, weil exakt darin der praktische Zweck des bulgarischen Aorists zu sehen ist, und sie ist bei Weitem nicht neu und wurde in der Bulgaristik schon 
ausschweifend diskutiert. Ich verweise nur auf die Grammatiktradition an unserer Universität, die maßgeblich von Professor Ivan Kucarov geprägt wurde. Dieser beschreibt in seiner über 600 Seiten starken "Theoretischen Grammatik der bulgarischen Sprache" ganz eindeutig, dass die eigentliche und vor allem die einzige echte Vergangenheitsform des Bulgarischen der Aorist ist (Куцаров 2007: 244-246).

Die Mitherausgeberin Nelli Ritter beschreibt anschließend „Leseindikatoren im Russischen und Deutschen: Eine Eye-TrackingStudie mit Herkunftssprecher/innen und lebensweltlich Monolingualen" (S. 209-228). Ihr Aufsatz thematisiert die Resultate ihrer Forschungen zu Lesekompetenzen im Deutschen und im Russischen. An der dokumentierten Untersuchung beteiligten sich demnach deutschrussisch bilinguale Sprecher im Alter zwischen 15 und 16 Jahren und gleichaltrige monolinguale Personen. Im Ergebnis zeigen die beiden Gruppen untereinander gravierende Kompetenzunterschiede. Ritter stellt selbst erhobene Daten vor und wertet sie kritisch aus. Das Thema und die gewinnbringende Interpretation sind sehr sinnvoll, um die Lesekompetenzen und die Bedürfnisse unterschiedlicher Sprechergruppen zu beschreiben und fundiert zu erforschen. Durchdacht wirkt auch der Ansatz, die in Deutschland weit verbreitete deutsch-russische Zweisprachigkeit in den Fokus zu rücken, schließlich handelt es sich nun einmal um eine Ressource, welche man sich vor allem in der Slavistik zu eigen machen kann.

Ksenija Vossmiller hinterfragt in ihrem Artikel "Quo vadis, Studierende der Herkunftssprache Russisch?" auf den Seiten 229 bis 242 die Bedeutung von Studenten mit Migrationshintergrund. Über diese Gruppe ist im Hinblick auf die Wahrnehmung ihrer russischen Herkunftssprache kaum etwas bekannt, und erst recht ist es selten, dass eine Unterteilung in Herkunftssprecher und andere, die zwecks Spracherwerbes eine deutsche Universität aufsuchen, überhaupt stattfindet. Sie attestiert daher der Beschäftigung mit der Herkunftssprache ein wichtiges Potential, wobei gerade in Deutschland auch ein recht hoher Assimilationsdruck an die deutsche Sprache existiert, so dass zu hoffen bleibt, dass diese Konstellation nicht auf lange Sicht zu einem Sprachverlust führt.

Valeria Wagner schreibt „Zum Altersdiskurs: Bezeichnungen von Älteren in Deutschland, Russland und bei russischsprachigen Migrant/innen im Vergleich“ (S. 243-266). Hier geht es um den Diskurs, der sich mit der Frage nach dem Alter von Menschen und den dazugehörigen Eigenschaften befasst. Die Autorin hinterfragt, wie dieser Lebensabschnitt in Zeitungen der russischen, der deutschen und der russlanddeutschen Gemeinschaft benannt wird. Um dem 
nachzugehen, stellt sie einen aus über 1300 Zeitungsartikeln bestehenden Korpus zusammen. Dieser Umfang zeigt nicht nur, wie relevant die Fragestellung ist, sondern auch, wie man sie in ein kontrastives Licht rücken kann.

Der letzte Aufsatz stammt von Veronika Wald und trägt den Titel „Verbvalenzstrukturen des Russischen und des Deutschen bei RussischHerkunftssprechern in Deutschland" (S. 267-290). Es werden die Valenzstrukturen untersucht, die die Autorin eigenen Angaben zu Folge bei bilingualen Sprechern des Deutschen und des Russischen in der BRD beobachten konnte. Besonders interessiert sie dabei, welche Valenzabweichungen der Verben sich im Sprachgebrauch experimentell beobachten lassen und worauf diese zurückzuführen sind. Der Beitrag zeigt, dass es interessante Valenzstrukturen gibt, die durch den deutschrussisch geprägten sprachlichen Alltag der Teilnehmer zu erklären sind. Man könnte gewiss noch stärker betonen, dass sie das Ergebnis eines alläglichen Sprachkontakts und daher zu erwarten sind. Das Phänomen wird aber gut von Wald beschrieben und nachvollziehbar begründet.

Am Ende möchte ich einige persönliche Bemerkungen anführen. Der vorliegende Band zeigt, dass deutsche Nachwuchswissenschaftler darum bemüht sind, sich systematisch zu organisieren und ihre Themen in der Slavistik zu präsentieren. Vor allem ist dies daran erkennbar, dass die Organisatoren finanzielle Unterstützung von der „Hamburgischen Wissenschaftlichen Stiftung" (HWS) und der "Deutsch-bulgarischen Gesellschaft zur Förderung der Beziehungen zwischen Deutschland und Bulgarien e.V." (DBG) erhielten und auf diese Weise ihr Werk in einem prestigereichen Verlag publizieren konnten (wovon wir im einleitenden Vorwort erfahren). Auch inhaltlich wecken die Beiträge das Interesse des Betrachters, denn sie sind anspruchsvoll geschrieben und thematisieren unterschiedliche slawische Sprachen. Außerdem verfolgen sie originelle Ansätze, die sie innovativ mit den neuesten Erkenntnissen aus dem Bereich der Slavistik verbinden. Die jungen Slavisten machen sich große Mühe bei der Erstellung eigener Korpora, sie sammeln Daten, konzipieren originelle empirische Studien, stellen eigene Theorien auf und setzen sich kritisch mit vorhandenem Wissen auseinander. Den Herausgebern ist es gelungen, interessantes Material zusammenzustellen und der Wissenschaft zugänglich zu machen. Der Sammelband kann aufgrund seiner überzeugenden Inhalte ohne jeden Zweifel der wissenschaftlichen Öffentlichkeit empfohlen werden und setzt wertvolle Akzente im Rahmen der slavistischen Linguistik, die ein hohes Potential für die Zukunft erkennen lassen. 


\section{FOLIA LINGUISTICA ET LITTERARIA:}

Literatur

Куцаров, Иван. Теоретична граматика на българския език. Морфология. Пловдив: ПУ Паисий Хилендарски, 2007. 